\title{
Apoptosis inhibitor TRIAP1 is a novel effector of drug resistance
}

\author{
CAROLINE ADAMS $^{1 *}$, GIULIA CAZZANELLI ${ }^{*}{ }^{*}$, SABEENA RASUL $^{1}$, BEN HITCHINSON $^{1}$, YUNHUI HU $^{1,2}$, \\ R. CHARLES COOMBES ${ }^{1}$, SELINA RAGUZ ${ }^{3}$ and ERNESTO YAGÜE ${ }^{1 *}$ \\ ${ }^{1}$ Cancer Research Centre, Division of Cancer, Imperial College London, Hammersmith Hospital Campus, \\ London W12 0NN, UK; ${ }^{2}$ Department of Breast Cancer, China Tianjin Breast Cancer Prevention, Treatment \\ and Research Center, Tianjin Medical University Cancer Institute and Hospital, Tianjin 300020, P.R. China; \\ ${ }^{3}$ Institute of Clinical Sciences, Imperial College London, Hammersmith Hospital Campus, London W12 0NN, UK
}

Received February 20, 2015; Accepted March 23, 2015

DOI: $10.3892 /$ or.2015.3988

\begin{abstract}
TP53-regulated inhibitor of apoptosis 1 (TRIAP1) is a novel apoptosis inhibitor that binds HSP70 in the cytoplasm and blocks the formation of the apoptosome and caspase- 9 activation. TRIAP1 has been shown to be upregulated in many types of cancers; however, its role remains elusive. We determined the TRIAPI mRNA levels in a panel of human tissues and found its expression to be ubiquitous. Normal breast, as well as non-tumorigenic breast cells, exhibited lower TRIAPI mRNA levels than breast cancer cells or their drug-resistant derivatives. TRIAP1 is a small, evolutionarily conserved protein that is 76 amino acids long. We found that yeast cells, in which the TRIAPI homologue was knocked out, had increased sensitivity to doxorubicin. Equally, RNA interference in breast cancer drug-resistant cells demonstrated that downregulation of TRIAP1 impaired cell growth in the presence of doxorubicin. As expected, caspase-9 activation was diminished after overexpression of TRIAP1 in drug-resistant cells. Importantly, stable transfections of a TRIAP1 expression plasmid in CAL51 cells led to a marked increase in the number of doxorubicin-resistant clones, that was abolished when cells expressed hairpins targeting TRIAP1. In addition, we showed that TRIAP1 expression was also triggered by estrogen deprivation in MCF-7 cells. Although both polyclonal and
\end{abstract}

Correspondence to: Dr Ernesto Yagüe, Cancer Research Centre, Division of Cancer, Imperial College London, Hammersmith Hospital Campus, Du Cane Road, London W12 0NN, UK

E-mail: ernesto.yague@imperial.ac.uk

"Contributed equally

Abbreviations: Apaf-1, apoptotic protease activating factor 1; DMEM, Dulbecco's modified Eagle's medium; DMEM-PR, DMEM lacking phenol red; DSS, dextran-coated charcoal-stripped FCS; FCS, foetal calf serum; HMECs, human primary mammary epithelial cells; IAP, inhibitor of apoptosis; TRIAP1, TP53-regulated inhibitor of apoptosis 1

Key words: inhibitors of apoptosis, drug resistance, doxorubicin, caspases, estrogen, tamoxifen monoclonal antibodies generated for the present study failed to robustly detect TRIAP1, we demonstrated that TRIAP1 represents a novel marker for drug resistance in breast cancer cells and it may be used in the stratification of breast cancer patients once a suitable antibody has been developed. Equally, these studies open potential drug development strategies for blocking TRIAP1 activity and avoiding drug resistance.

\section{Introduction}

One of the many mechanisms by which cancer cells acquire drug resistance is by apoptosis evasion $(1,2)$. Antineoplastic agents activate the apoptotic intrinsic pathway which results in the permeabilization of the outer mitochondrial membrane. The subsequent release of cytochrome $c$ and other pro-apoptotic molecules leads to the formation of a large protein complex, the apoptosome, containing cytochrome $c$, apoptotic protease activating factor 1 (Apaf-1) and caspase-9. Regulation of these processes occurs through the activity of members of the BCL-2 family of proteins (3). Upregulation of anti-apoptotic proteins, such as BCL-2, BCL-XL, and members of the inhibitor of apoptosis (IAP) family, and inactivating mutations in pro-apoptotic proteins Bax and Bak, or p53 have been described in both the development of cancer and drug resistance $(2,4)$. Among several therapeutic strategies, the restoration of p53-mediated apoptosis in cancer cells has been sought for some time, so far without a clinical application $(5,6)$. Therefore, targeting inhibitors of apoptosis offer another opportunity for drug development (7).

TP53-regulated inhibitor of apoptosis 1 (TRIAP1; alias p53CSV, p53-inducible cell-survival factor) is a small, evolutionarily conserved protein that is 76 amino acids long and contains a twin $\mathrm{Cx}_{9} \mathrm{C}$ motif. Its expression is induced by $\mathrm{p} 53$ following low levels of genotoxic stress (8). In yeast it is located in the mitochondrial intermembrane space where it can interact with both Ups1 and Ups2, protecting them against proteolysis and regulating cardiolipin and phosphatidylethanolamine levels within mitochondria (9). In its extramitochondrial role, TRIAP1 modulates apoptotic pathways through interaction with HSP70, inhibition of the interaction of cytochrome $c$ with Apaf-1 and activation of caspase-9, to inhibit apoptosis and permit DNA damage repair $(8,10)$. Despite its name, TRIAP1 
does not share any similarity with XIAP, c-IAP, survivin or other members of the IAP family (11). TRIAPI is upregulated in myelomas with unfavorable prognosis (12) and TRIAPI mRNA has been found in all breast cancer cell lines tested (13). However, the role of TRIAP1 in breast or other solid tumors has been poorly addressed.

Breast cancer is one of the most common tumors affecting women, and chemotherapy, endocrine therapy and radiotherapy regimens are part of the present mainstream treatment. Even those cancers that initially respond well to treatment may become resistant and give rise to secondary tumors, normally with fatal consequences (14). Thus, it is crucial to be able to predict which patients will respond to therapy in order to design the best clinical strategy available and to develop drugs for the treatment of resistance (15).

Here we describe the upregulation of TRIAP1 in drug-resistant breast cancer cells. Experimental modulation of TRIAP1 in breast cancer cells, either by overexpression or downregulation by RNA interference, changed cellular sensitivity to doxorubicin, thus confirming TRIAP1 as a novel effector of drug resistance.

\section{Materials and methods}

Cell culture, transduction and transfection. The breast cancer cell lines CAL51 (German Resource Centre for Biological Material, DSMZ, Braunschweig, Germany) and MCF7 (European Collection of Cell Cultures, Health Protection Agency, UK) were grown in low glucose (1 g/l) Dulbecco's modified Eagle's medium (DMEM)-GlutaMax (Invitrogen, Carlsbad, CA, USA) supplemented with $10 \%$ foetal calf serum (FCS). Drug-resistant derivatives CALDOX, MCFDOX and MLET5 have been previously described (16-18). RNA from MDA-MB-231, T47D, ZR75 and 226-L-U19 cells (19) was a generous gift from Y. Zhou. Human primary mammary epithelial cells (HMECs) were purchased from Invitrogen and immortalized by expression of the catalytic subunit of human telomerase as previously described (20) to generate HMEC-TERT cells. HMECs were grown on Mammary Epithelial Cell Growth Medium (Invitrogen). GP2-293 pantropic retroviral packaging (Clontech Laboratories, Mountain View, CA, USA) and HEK293T cells (American Type Culture Collection, LGC Standards, Teddington, UK) were maintained in DMEM supplemented with $4.5 \mathrm{~g} / 1$ glucose, $10 \%$ FCS and $4 \mathrm{mM}$ L-glutamine (Invitrogen). For culturing in estrogen-free conditions, MCF-7 cells were cultured in DMEM lacking phenol red (DMEM-PR; Gibco-BRL, UK), supplemented with $10 \%$ dextran-coated charcoal-stripped FCS (DSS; First Link Ltd., UK). MLET5 cells were routinely cultured in DMEM-PR, containing 10\% DSS. All cells were routinely tested for the presence of mycoplasma using the Lonza MycoAlert kit (Basel, Switzerland).

DNA procedures. A human TRIAP1 cDNA clone with a C-terminal GFP tag cloned into pCMV6-AC-GFP was purchased from Origene and used for transient transfections. For stable transfections, the genomic region encompassing TRIAPI (first and second exons including both 5'- and 3'-UTRs as well as the single intervening sequence) was amplified from genomic DNA isolated from IMR-90 fibroblasts by PCR using
Platinum ${ }^{\circledR}$ Taq DNA Polymerase High Fidelity (Invitrogen) with primers OLEY335 and OLEY336 (Table I). The amplified 2.6-kbp fragment was initially cloned into pCRII-TOPO (Invitrogen) and the TRIAPI open reading frame verified by sequencing. Then, the genomic TRIAP1 fragment was liberated by digestion with BamHI and EcoRI and cloned into the BamHI- and EcoRI-digested lentiviral vector FUW. Retroviral plasmids pGFP-V-RS expressing short hairpins targeting TRIAP1 (5'-AGAGATTCCTATTGAAGGACTGG AGTTCA-3') and non-targeting scrambled (TR30013) were from Origene.

Viral transduction. Viral transductions were essentially as previously described (16). Briefly, retroviral transfections were performed using $20 \mu \mathrm{g}$ retroviral plasmid, $2 \mu \mathrm{g}$ pVSV-G (Clontech Laboratories) and $55 \mu \mathrm{g}$ Polyethylenimine (MW 25,000) (Polysciences, Warrington, PA, USA) prior to co-transfection into the GP2-293 pantropic retroviral packaging cells. Lentiviral transfections were performed using $20 \mu \mathrm{g}$ lentiviral plasmid, $2 \mu \mathrm{g}$ pVSV-G, $8 \mu \mathrm{g}$ pPAX2 (Addgene, Cambridge, MA, USA) and $75 \mu \mathrm{g}$ Polyethylenimine (MW 25,000) (SigmaAldrich, St. Louis, MO, USA) prior to co-transfection into the HEK293T cells. Viral supernatants were collected 24-48 h after transfections, filtered through $0.45-\mu \mathrm{m}$ cellulose acetate filters and supplemented with $8 \mu \mathrm{g} / \mathrm{ml}$ Polybrene (SigmaAldrich) prior to adding to recipient cells. Puromycin $(1 \mu \mathrm{g} /$ $\mathrm{ml}$; Sigma-Aldrich) was added $96 \mathrm{~h}$ after the first infection to recipient cells for selection of stable transgene expression.

Transient transfections. Cells $\left(1 \times 10^{5}\right.$ in 6 -well plates) were transfected with a final concentration of $40 \mathrm{nM}$ ON-TARGETplus SMARTpool TRIAPI siRNA oligonucleotides (Thermo Scientific) using HiPerFect (Qiagen, UK) following the manufacturer's instructions. EGFP siRNA (Ambion, UK) was used as a negative control (21). A sulphorhodamine B (Sigma-Aldrich) assay (22) was used to screen for drug cytotoxicity as previously described (19). For transient overexpression, cells $\left(1 \times 10^{4}\right)$ were seeded in 96-well plates and transfected with FuGENE HD (Promega, Madison, WI, USA) using $2 \mu \mathrm{g}$ DNA as recommended by the manufacturer. Then cells were treated for $24 \mathrm{~h}$ with $0.4 \mu \mathrm{M}$ doxorubicin, and caspase- 9 activity was determined using a Caspase-Glo 9 assay (Promega) following the manufacturer's instructions and normalized to cell density obtained after sulphorhodamine B staining.

Gene expression analysis. Total RNA (isolated using RNAzol B; Biogenesis, Poole, UK) was reverse transcribed with RNase $\mathrm{H}^{+}$MMLV Reverse Transcriptase (iScript cDNA Synthesis kit; Bio-Rad) and real-time quantitative PCR was performed using SYBR-Green (Bioline Reagents, London, UK) and gene specific primers (Table I) on an ABI Prism 7700 Detection system (PerkinElmer Life Sciences, Waltham, MA, USA). PCR conditions included an initial step at $95^{\circ} \mathrm{C}$ for $10 \mathrm{~min}$ followed by 40 cycles of $95^{\circ} \mathrm{C}$ for $30 \mathrm{sec}, 60^{\circ} \mathrm{C}$ for $30 \mathrm{sec}$ and $72^{\circ} \mathrm{C}$ for $30 \mathrm{sec}$. A comparative threshold cycle was used to determine the relative gene expression as previously described (23). TFF1 expression was performed using TaqMan Gene Expression assay Hs00907239_m1 (Invitrogen) following the manufacturer's instructions. 
Table I. Oligonucleotides used in this study,

\begin{tabular}{lll}
\hline Primer & \multicolumn{1}{c}{ Sequence (5'-3') } & \multicolumn{1}{c}{ Use } \\
\hline OLEY335 & GGGGGATCCCGACGCGCCTGAGAGTGATGACATCA & TRIAP1 genomic amplification \\
OLEY336 & GGGGTCGACTGTGAGGTTTCTGATTGCCATACTG & TRIAP1 genomic amplification \\
OLEY287 & AGGATTTCGCAAGTCCAGAA & TRIAP1 QPCR \\
OLEY288 & GCTGATTCCACCCAAGTAT & TRIAP1 QPCR \\
OLEY373 & TCACCGCCCTACACATCAAACT & RPS14 QPCR \\
OLEY374 & CTGCGAGTGCTGTCAGAGG & RPS14 QPCR \\
OLEY375 & AGGGTTATGTGGTCCGAATCA & RPS6 QPCR \\
OLEY376 & TGCCCCTTACTCAGTAGCAGG & RPS6 QPCR \\
\hline
\end{tabular}

Yeast procedures. Wild-type Saccharomyces cerevisiae

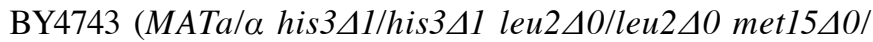
MET15 lys2 $\Delta 0 /$ LYS2 ura3 0 /ura3 $\Delta 0$ ) and Mdm35 knockout strain were purchased from Open Biosystems. Screening for drug toxicity was performed in YPD plates with doxorubicin after 3 days of growth.

Drug resistance clonogenic assay. Cells were seeded, at least in duplicate, at a density of $1-5 \times 10^{5}$ cells in $25-\mathrm{cm}^{2}$ culture flasks and exposed to a single dose of doxorubicin for $24 \mathrm{~h}$. Cells were kept in culture for 2-4 weeks with drug-free medium changes every week. Drug-resistant clones were fixed with $4 \%$ paraformaldehyde and stained with $0.2 \%$ crystal violet. Crystal violet retained in the cells was quantified by solubilization with $33 \%$ acetic acid and measured at $\mathrm{OD}_{592} \mathrm{~nm}$.

Statistical analysis. Statistical evaluations were performed using the Student's t-test for paired data, and data were considered significant at a P-value $<0.05$.

\section{Results}

TRIAP1 is ubiquitously expressed in normal human tissues and upregulated in breast cancer cells. We initially tested TRIAP1 expression in a panel of RNAs from normal human tissues (24) and found it ubiquitously expressed (Fig. 1A). Although there was a lack of tissue specificity, bone marrow, heart and kidney showed very low expression, whereas brain, placenta and testis showed high expression, with that from skeletal muscle being the highest. Normal breast tissue expressed TRIAP1 mRNA at intermediate levels (Fig. 1A). Next we tested TRIAPI mRNA expression in four breast cancer cell lines (CAL51, MDA-MB-231, T47D and ZR75) and in two immortal, but non-tumorigenic, breast cell lines (HMEC-TERT and 226-L-U19). An 2-fold upregulation was found in the cancerous cell lines, indicating that malignant transformation of breast epithelial cells is, at least in vitro, associated with TRIAP1 upregulation (Fig. 1B).

A polyclonal antibody raised to the synthetic peptide CVQKAIKEKEIPIEGLEF (amino acids 47-64) as well as three monoclonal antibodies raised to the epitopes HGKEKPENSS (amino acids 67-76), IKEKEIPIEG (amino acids 52-61) and FAEKFLKGDS (amino acids 23-32), all failed to robustly detect TRIAP1 as a band at $\sim 10 \mathrm{kDa}$ (data not shown). Equally, a few commercial antibodies were tested and failed in the same way (data not shown). Thus, TRIAP1 expression analyses throughout the present study were performed exclusively at the mRNA level.

TRIAP1 is upregulated in drug-resistant breast cancer cell lines. TRIAP1 upregulation following genotoxic stress has been exclusively demonstrated in a colon cancer cell line (8). We confirmed that CAL51 breast cancer cells exhibited upregulation of TRIAPI mRNA expression after $24 \mathrm{~h}$ of doxorubicin treatment at low to moderate doses (up to $0.4 \mu \mathrm{M}$ ). Higher doses $(2-5 \mu \mathrm{M})$ repressed TRIAP1 expression (Fig. 1C). Then we examined whether TRIAP1 was associated with a drugresistance phenotype. For this, we tested TRIAPI mRNA expression in two doxorubicin-resistant breast cancer cell lines derived from CAL51 (16) and MCF7 (17) cells, and in a third cell line derived from MCF7 cells after estrogen deprivation, which are resistant to tamoxifen and etoposide (18). Doxorubicin and etoposide are two topoisomerase II inhibitors producing genotoxic stress, ultimately leading to apoptosis, which form part of many chemotherapeutic cancer regimes. TRIAPI mRNA was upregulated in these three drug-resistant cell types between 3- and 8-fold (Fig. 1D).

TRIAPI is an effector of drug resistance. In order to determine whether TRIAP1 is an effector of drug resistance, we tested cell susceptibility to doxorubicin after experimental modulation of TRIAP1 expression. Comparison of TRIAP1 protein sequences revealed a striking conservation across kingdoms (Fig. 2A), albeit not at the C-terminus, indicating that this protein must perform a fundamental role in cellular physiology. The TRIAP1 homologue in S. cerevisiae, Mdm35, shares $33 \%$ identity with human TRIAP1. Since yeast cells harbour an ancestral programmed cell death machinery that shares many key molecules that are part of the apoptotic intrinsic pathway in mammalian cells (25), and because genetic manipulation of yeast allows the knockout of genes, we initially tested our hypothesis in $M d m 35$ knockout yeast cells. Indeed, $\Delta M d m 35$ cell growth was greatly inhibited by the presence of doxorubicin in the yeast growth medium compared to wild-type cell growth (Fig. 2B).

Next, we determined whether experimental downregulation of TRIAP1 would have a similar effect in breast cancer cells. For this, we transiently transfected siRNAs targeting TRIAPI 

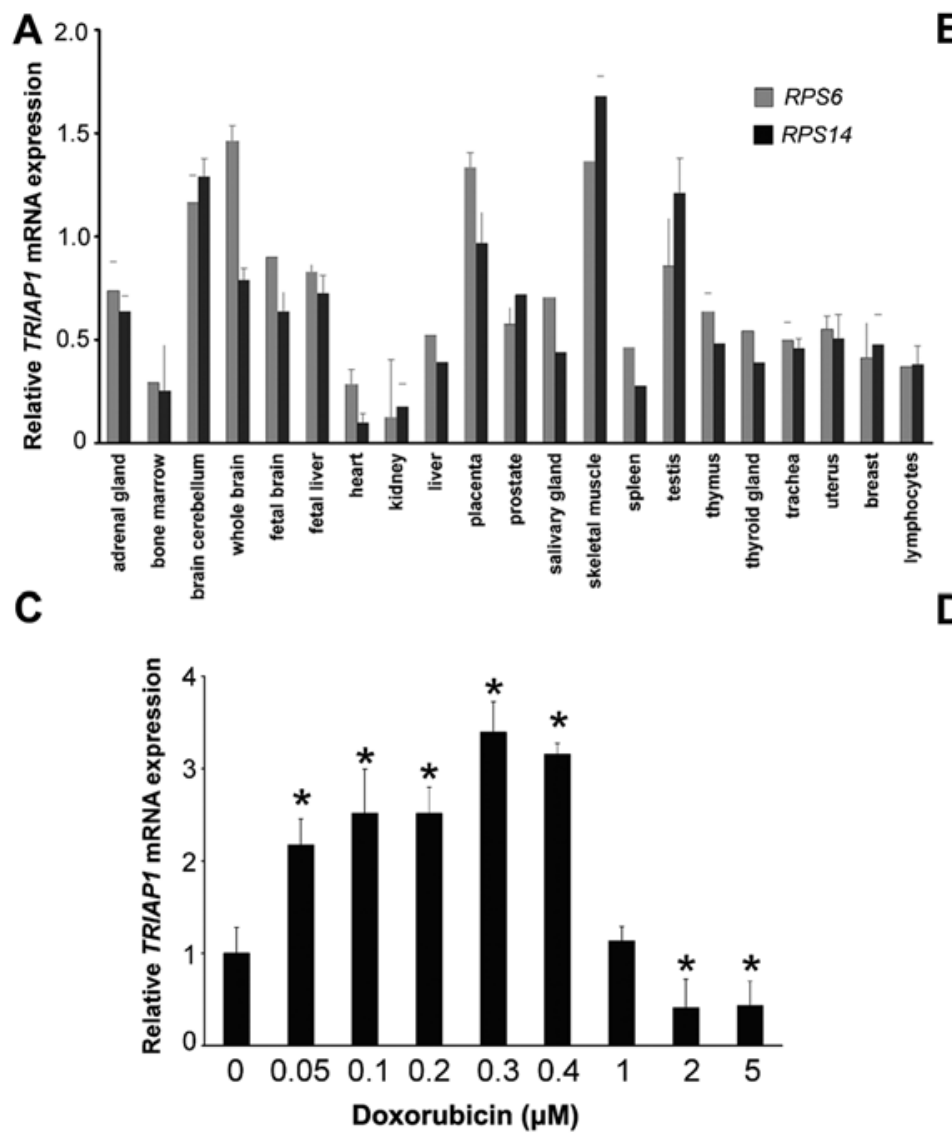

B

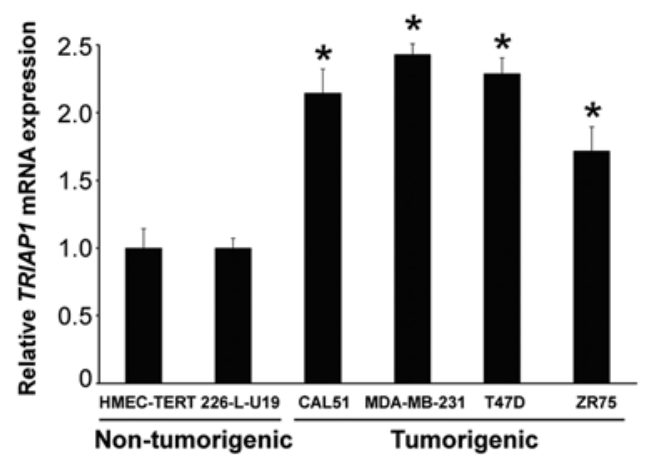

D

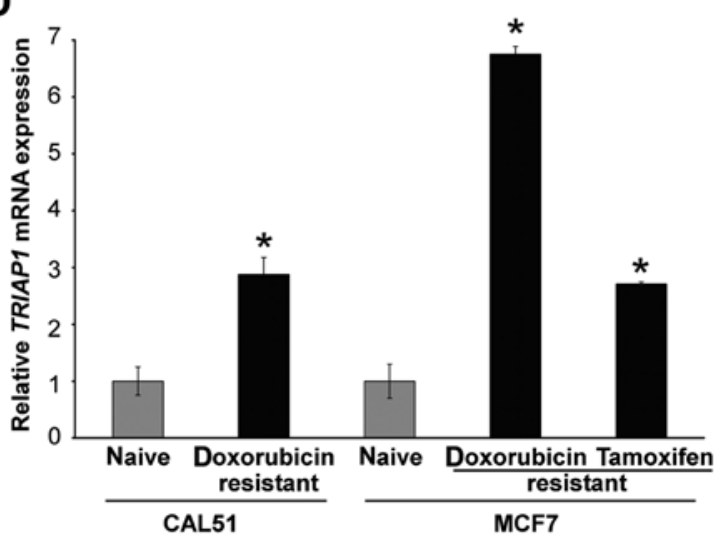

Figure 1. TRIAP1 is a ubiquitously expressed gene which is upregulated in transformed mammary epithelial cells, after genotoxic stress, and in drug-resistant cells. (A) TRIAP1 mRNA is expressed ubiquitously in normal human tissues. A panel of 21 human tissues was used to analyze TRIAP1 mRNA expression by RT-QPCR. Two normalizers, RPS6 and RPS14 were used. (B) TRIAP1 mRNA is upregulated in tumorigenic breast cancer cell lines. A small panel of both tumorigenic and non-tumorigenic breast cell lines was used to determine TRIAP1 mRNA expression by RT-QPCR. Data were normalized to RPS6 expression. (C) TRIAP1 gene expression is upregulated after moderate genotoxic stress. Breast cancer CAL51 cells were treated with doxorubicin for $24 \mathrm{~h}$ and TRIAP1 mRNA expression was determined by RT-QPCR. Data were normalized to RPS6 mRNA expression. (D) TRIAP1 mRNA expression is upregulated in drug-resistant breast cancer cells. TRIAP1 mRNA expression was determined by RT-QPCR and normalized to RPS6. Doxorubicin-resistant CALDOX and MCFDOX cells were derived from CAL51 and MCF-7, respectively. MLET5 is an estrogen-deprivation resistant derivative from MCF-7 cells with resistance to tamoxifen. Data represent the average $\pm \mathrm{SD}$ of three independent experiments $\left({ }^{*} \mathrm{P}<0.05\right)$.

mRNA in doxorubicin-resistant CALDOX cells and obtained $\sim 60 \%$ downregulation in TRIAP1 mRNA levels (Fig. 3A). Transfection of siRNAs targeting EGFP mRNA, which is not expressed in these cells, was used as a negative control. This transient decrease in TRIAPI mRNA levels was sufficient to inhibit the proliferation of cells in the presence of doxorubicin compared to the slight decrease in proliferation obtained with the negative control (Fig. 3B).

As TRIAP1 blocks the formation of the apoptosome and activation of caspase-9 (8), we next ascertained whether caspase- 9 activity would be reduced by TRIAP1 in breast cancer cells. To do this, we transiently overexpressed TRIAP1 in CAL51 cells (Fig. 3C) and treated them with $0.4 \mu \mathrm{M}$ doxorubicin for $24 \mathrm{~h}$. As expected, the control cells transfected with an empty vector exhibited increased caspase-9 activity, and this increase was reduced in the cells overexpressing TRIAP1 (Fig. 3D).

Although experiments performed with transient transfections are informative to assess the short-term susceptibility to cytotoxic drugs, the generation of drug resistance is a slower process, often taking several weeks in vitro, and required a different approach. We generated CAL51 derivative cells that had either permanently downregulated TRIAP1 by stable expression of hairpins targeting TRIAPl mRNA (shTRIAP) or permanently upregulated TRIAP1. CAL-shTRIAP cells showed decreased levels of TRIAP1 mRNA by $\sim 60 \%$ (Fig. 4A). In addition, we managed to reproduce the slight increase in TRIAPI mRNA expression found in drug-resistant CALDOX cells by using low multiplicity of infection of the lentivirus used for TRIAP1 overexpression (Fig. 4A). Thus, although these experimental changes in TRIAP1 expression after stable gene expression were modest, they reflect very accurately the difference found between naïve and drug-resistant cells (Fig. 1D). Next we performed clonogenic assays after doxorubicin treatment. For this, doxorubicin was added to cell cultures and the emergence of doxorubicin-resistant clones was monitored over a period of 2-4 weeks. Stable experimental overexpression of TRIAP1 led to an increase in doxorubicin-resistant clones, and, conversely, downregulation of TRIAP1 led to fewer drug-resistant clones than the control cells (Fig. 4B and C).

TRIAPI is upregulated by lack of estrogen. As tamoxifen-resistant cells have elevated levels of TRIAPI mRNA (Fig. 1D), 


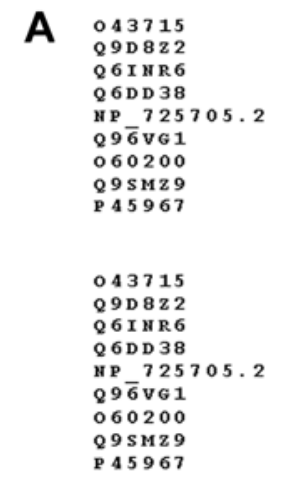

B
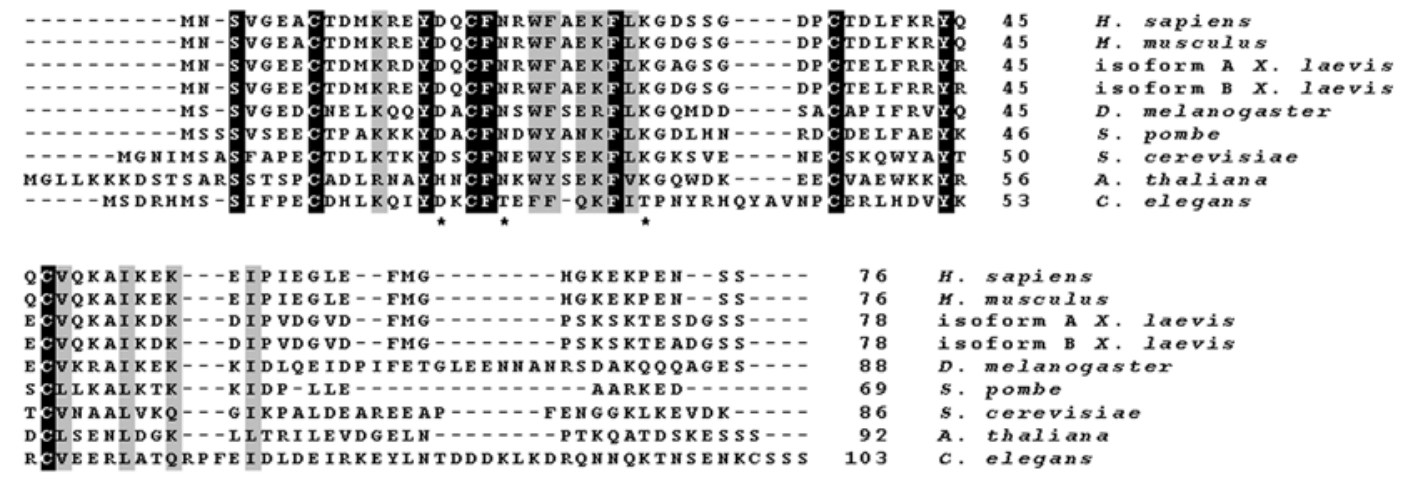

H. Sapiens
H. musculus

isoform A X laevis

is oform B $X$. laevis

D. melanogaster

s. pombe

s. cerevisiae

A. thaliana

c. elegans

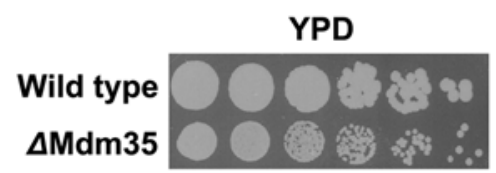

YPD + doxorubicin $(\mu \mathrm{g} / \mathrm{ml})$

$\begin{array}{lll}12.5 & 25.0 & \mathbf{5 0 . 0} \\ & 0 & 0\end{array}$

Figure 2. Knockout of TRIAP1 in yeast leads to increased doxorubicin sensitivity. (A) TRIAP1 is a highly conserved protein. TRIAP1 protein sequences (accession numbers indicated on the left) of several organisms (indicated on the right) were aligned using ClustaLW at the European Bioinformatics Institute. Identical amino acids are shown in black background and similar ones in grey background. Conserved amino acids with a lesser degree of similarity are indicated with an asterisk. (B) Saccharomyces cerevisiae cells with the TRIAPI homologue Mdm35 knocked out are sensitive to doxorubicin. Yeast cell cultures at different dilutions were spotted onto YPD plates with or without doxorubicin and left to grow for 3 days.

A
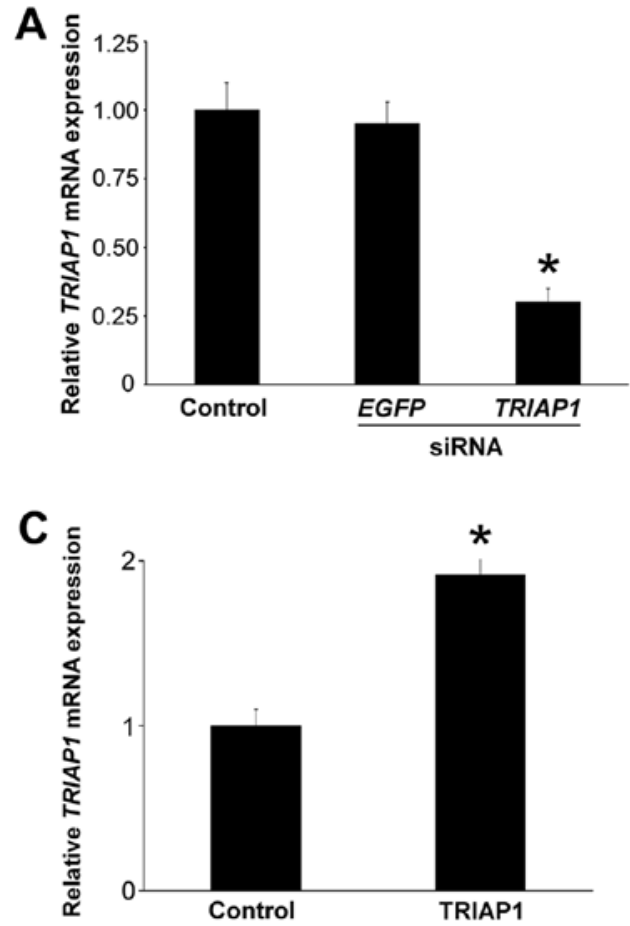

B

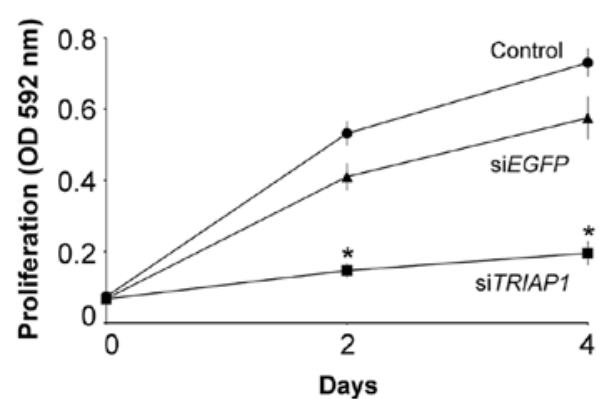

D

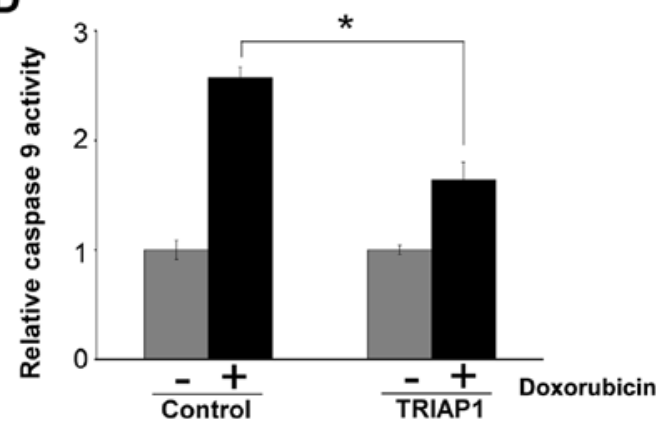

Figure 3. Transient modulation of TRIAP1 expression affects the sensitivity of breast cancer cells to doxorubicin. (A and B) Transient downregulation of TRIAP1 increases drug sensitivity. (A) Doxorubicin-resistant CALDOX cells were transiently transfected with siRNAs targeting either TRIAP1 mRNA or EGFP (used as negative control), and TRIAP1 mRNA expression was determined by RT-QPCR after normalization to RPS6. (B) After siRNA transfection, cell growth in the presence of $0.4 \mu \mathrm{M}$ doxorubicin was monitored (sulforhodamine B staining) for up to 4 days. (C and D) Transient overexpression of TRIAP1 blocks caspase-9 activation. Breast cancer CAL51 cells were transiently transfected with a TRIAP1 expression vector. (C) TRIAP1 mRNA levels were determined as above and (D) caspase-9 activation was determined $24 \mathrm{~h}$ after adding $0.4 \mu \mathrm{M}$ doxorubicin. Caspase-9 levels were normalized to cell density by sulforhodamine B staining. Data represent the average \pm SD of three independent experiments ( $\mathrm{P}<0.05)$.

we investigated whether a link exists between estrogen and TRIAP1 expression. We cultured MCF-7 and MLET5 cells for
3 days in estrogen-deprived medium, re-introduced estrogen for $16 \mathrm{~h}$, and then determined TRIAPl mRNA levels. There 
A

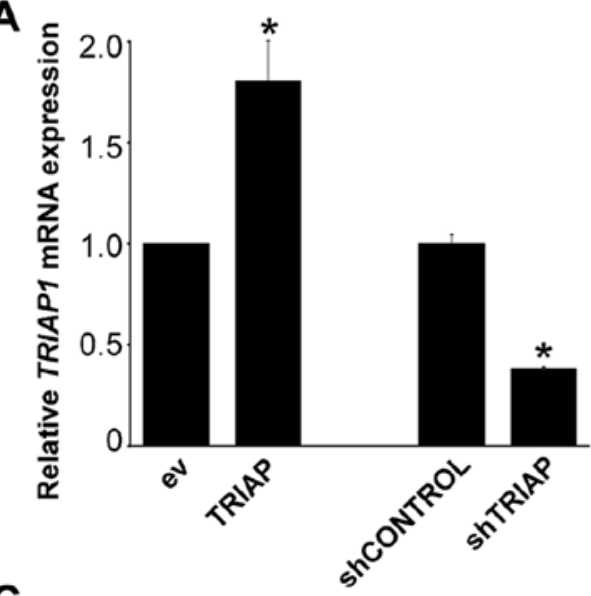

C

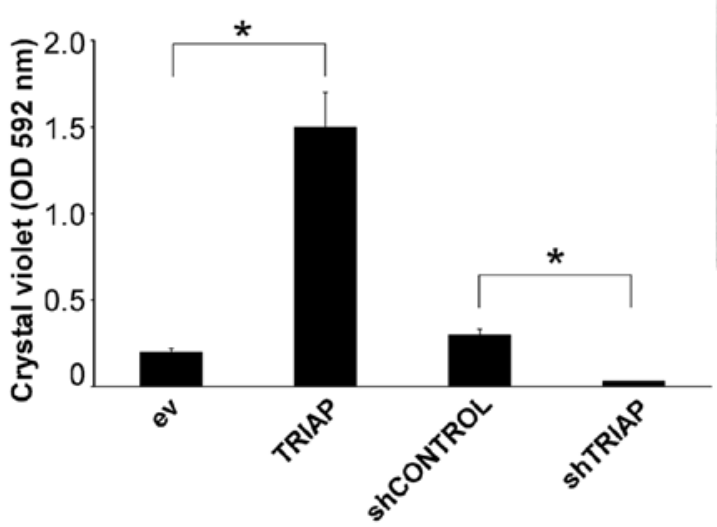

B

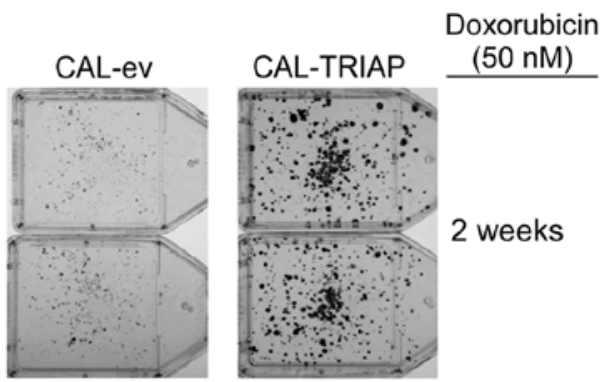

CALShCONTROL

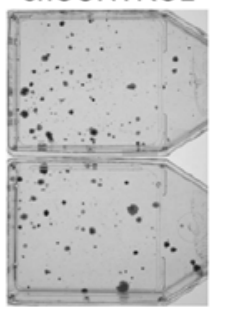

CAL-

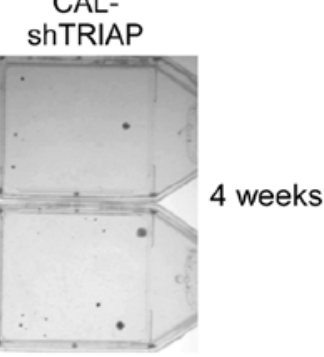

Figure 4. TRIAP1 is an effector of drug resistance. (A) CAL51 cells were stably transfected with a TRIAP1 expression construct (CAL-TRIAP) or empty vector as control $(C A L-e v)$. Stably transfected cells with either downregulated or upregulated TRIAP1 were generated by retroviral (small hairpins) or lentiviral transfection of CAL51 cells. TRIAPI mRNA expression was determined by RT-QPCR after normalizing to RPS6. (B) Cells $\left(10,000 / 25 \mathrm{~cm}^{2}\right)$ overexpressing TRIAP1 (CAL-TRIAP) (or empty vector control cells, $C A L-e v$ ) were treated with $50 \mathrm{nM}$ doxorubicin/ml and left in culture for 2 weeks (upper panel). Cells $\left(50,000 / 25 \mathrm{~cm}^{2}\right)$ expressing a hairpin targeting TRIAPI mRNA (CAL-shTRIAP) (or scrambled control cells, CAL-shCONTROL) were treated with $50 \mathrm{nM}$ doxorubicin $/ \mathrm{ml}$ and left in culture for 4 weeks (lower panel). Drug-resistant clones were stained with crystal violet. (C) Crystal violet was solubilized and optical density was determined at $592 \mathrm{~nm}$. Numerical data represent the average \pm SD of 3 experiments $($ ( $\mathrm{P}<0.05)$. Images show a representative of at least 3 replicates.

was no upregulation of TRIAP1 in any cell type; in fact, MCF-7 cells treated with $10 \mathrm{nM}$ estrogen showed a slight decrease in TRIAP1 expression (Fig. 5A). As expected, the estrogen-responsive gene TFF1 (26) was upregulated in both cell lines (Fig. 5A). This indicates that TRIAPl is not an estrogen-responsive gene. Importantly, when MCF-7 cells were cultured in the absence of estrogen, TRIAP1 expression was upregulated (Fig. 5B). Moreover, the addition of tamoxifen, which blocks estrogen receptor activation, led to a small but significant upregulation of TRIAPI mRNA in the MCF-7 cells, but not, as expected, in the tamoxifen resistant MLET5 cells (Fig. 5C).

Overall, these results establish TRIAPI as a novel effector of drug resistance in breast cancer cells.

\section{Discussion}

One of the many mechanisms by which cancer cells develop resistance to therapeutic intervention is by apoptosis evasion. This is not surprising since the apoptotic machine is complex, with multiple layers of regulation $(1,2)$. The IAP gene family encodes a group of structurally-related proteins sharing a BIR domain, such as XIAP or survivin, which can be upregulated in breast cancer $(27,28)$. However, other inhibitors of apoptosis do not share structural similarities with members of the IAP family. One such protein, TRIAP1, is a small, 76-amino acid protein in humans that binds to HSP70 and inhibits the formation of the apoptosome. Apoptosis evasion is one of the hallmarks of cancer (29), and is thus not surprising to find TRIAPI mRNA upregulated in a variety of malignancies (30-32) and in breast cancer cell lines, but not in TERT-immortalized primary mammary epithelial cells or in cells transformed but not yet fully tumorigenic (Fig. 1B). Although the mechanisms upregulating TRIAP1 during tumorigenesis are not known, a low to moderate amount of several genotoxic agents triggers its expression via p53 activation (8). Importantly we also found that estrogen deprivation upregulated TRIAP1. Estrogen deprivation reduces p53 activity $(33,34)$, and in normal mammary epithelial cells tamoxifen activates p53 (35), although not in MCF-7 cells (36). Thus, the upregulation of TRIAP1 in these cases is very likely to be p53-independent. Our data clearly demonstrated that TRIAP1 is a novel effector of drug resistance. Its experimental overexpression confers cells the capacity to develop doxorubicin-resistant derivatives, whereas its downregulation by RNA interference generates cells more susceptible to the cytotoxic effects of the drug. 

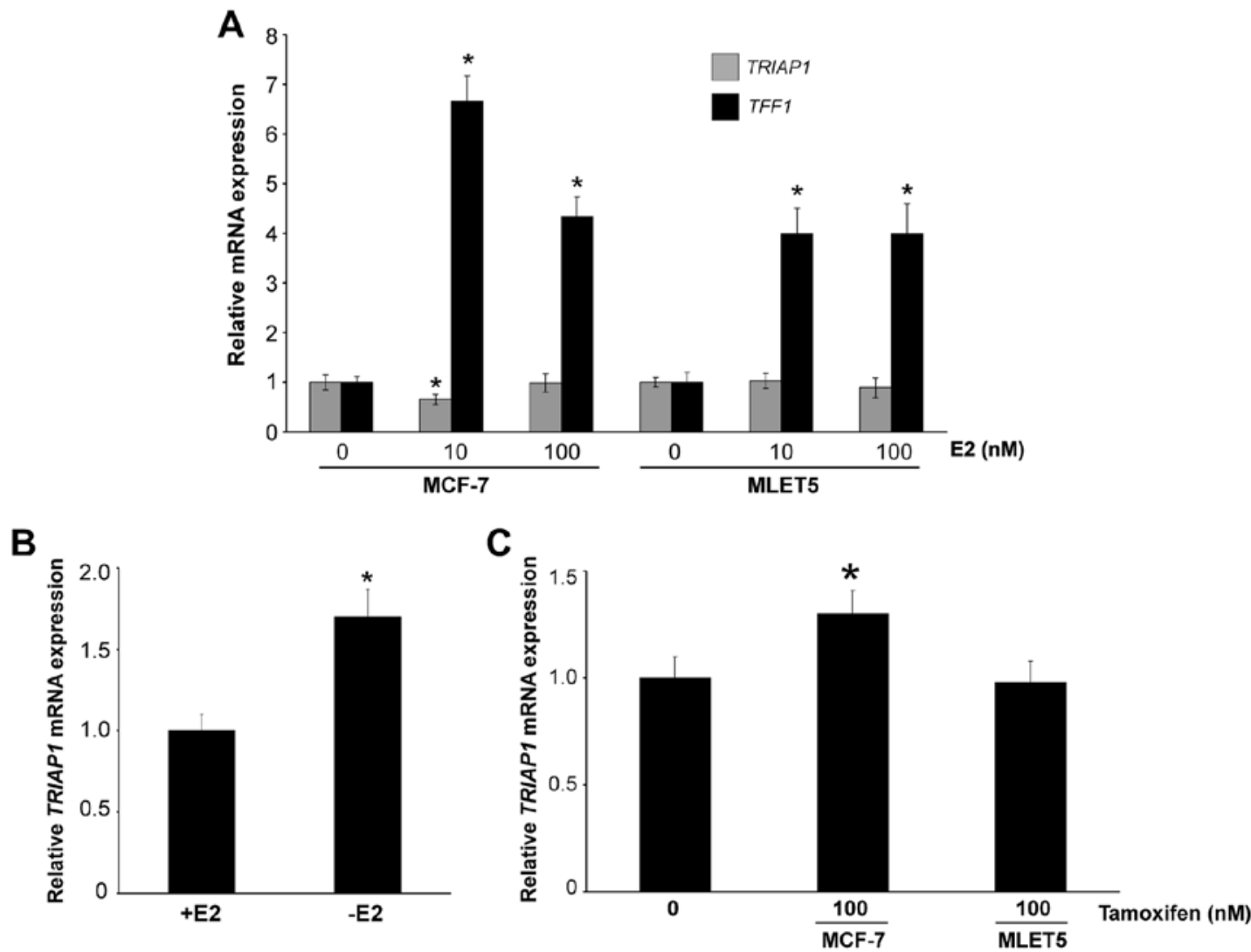

Figure 5. TRIAP1 is upregulated by estrogen deprivation. (A) TRIAP1 is not upregulated by estrogen. MCF-7 and MLET5 cells were deprived of estrogen for 3 days. Then $17 \beta$-estradiol (E2) was added to the medium, and the expression of TRIAP1 and TFF1 mRNAs was determined $16 \mathrm{~h}$ later by RT-QPCR after normalization to RPS6. (B) TRIAP1 is upregulated by estrogen deprivation. MCF-7 cells were grown for 3 days in medium containing (+E2) or deprived of (-E2) 17ß-estradiol, and the expression of TRIAP1 mRNA was determined by RT-QPCR. (C) TRIAP1 is upregulated by tamoxifen. MCF-7 and MLET5 cells were treated with $100 \mathrm{nM}$ tamoxifen for $48 \mathrm{~h}$, and the expression of TRIAP1 was determined by RT-QPCR. Expression is shown relative to the expression of each cell line in the absence of tamoxifen. Data represent the average \pm SD of 3 experiments ( $\mathrm{P}<0.05)$.

Due to its ubiquitous tissue expression in humans and extremely high conservation throughout evolution it is likely that TRIAP1 has an important function in eukaryotic cells. Research on yeast indicates that TRIAP1 (Mdm35) normally resides in the mitochondrial intermembrane space where it interacts with Usp1 and Usp2 (PRELI1 and PRELI2 in humans) to regulate the synthesis of cardiolipin and phosphatidylethanolamine, respectively (9). Although TRIAP1/PRELI complexes (Mdm35/Usp homologues in yeast) have been shown to prevent apoptosis by mediating intramitochondrial transport of phosphatidic acid in HeLa cells (37), it is tempting to speculate that the upregulation of TRIAP1 expression following moderate genotoxic stress (Fig. 1C), or that found in drug-resistant cells (Fig. 1D), helps to maintain the balance towards apoptosis inhibition by blocking apoptosome formation and caspase- 9 inactivation (8). However, after stronger genotoxic stress, which probably leads to irreparable DNA damage, there is no activation of TRIAP1 expression. A large escape of cytochrome $c$ from the mitochondria would be enough to change the balance towards apoptosome formation and the background levels of TRIAP1 protein would not be enough to block the interaction cytochrome $c$-Apaf1-caspase-9 (38). In addition, it is not clear whether, in addition to cytochrome $c$, TRIAP1 or TRIAP1/ PRELID complexes can also escape from the intermembrane space following stress to interact with HSP70. Nonetheless, yeast data as well as those presented here indicate a dual role for TRIAP1 in mitochondrial lipid biosynthesis and drug resistance. This may offer a potential drug development strategy for the synthesis of compounds blocking TRIAP1 activity.

\section{Acknowledgements}

We thank Yuan Zhou for a generous gift of MDA-MB-231, T47D, ZR75 and 226-L-U19 RNA, Hetal Dattani for advice on $T F F 1$ expression, Andres Clemente Blanco for advice on yeast work, and Eric Lam and Laki Buluwela for the MCFDOX and MLET5 cells, respectively. G.C. was the recipient of an Erasmus postgraduate scholarship. Y.H. was partially supported by the China Scholarship Council. We thank the Breast Cancer Research Trust (C.A.), Breast Cancer Campaign (2011MaySP01 to E.Y.), Cancer Research UK (R.C.C.) and the Experimental Cancer Medicine Centre Network (R.C.C.) for their financial support.

\section{References}

1. Mashima T and Tsuruo T: Defects of the apoptotic pathway as therapeutic target against cancer. Drug Resist Updat 8: 339-343, 2005.

2. Indran IR, Tufo G, Pervaiz S and Brenner C: Recent advances in apoptosis, mitochondria and drug resistance in cancer cells. Biochim Biophys Acta 1807: 735-745, 2011.

3. Elmore S: Apoptosis: a review of programmed cell death. Toxicol Pathol 35: 495-516, 2007. 
4. Cotter TG: Apoptosis and cancer: the genesis of a research field. Nat Rev Cancer 9: 501-507, 2009.

5. Yu Q: Restoring p53-mediated apoptosis in cancer cells: new opportunities for cancer therapy. Drug Resist Updat 9: 19-25, 2006

6. Kastan MB: Wild-type p53: tumors can't stand it. Cell 128 : 837-840, 2007.

7. de Graaf AO, de Witte T and Jansen JH: Inhibitor of apoptosis proteins: new therapeutic targets in hematological cancer? Leukemia 18: 1751-1759, 2004

8. Park WR and Nakamura Y: $p 53 C S V$, a novel p53-inducible gene involved in the p53-dependent cell-survival pathway. Cancer Res 65: 1197-1206, 2005.

9. Tamura Y, Iijima M and Sesaki H: Mdm35p imports Ups proteins into the mitochondrial intermembrane space by functional complex formation. EMBO J 29: 2875-2887, 2010.

10. Niu P, Liu L, Gong Z, Tan H, Wang F, Yuan J, Feng Y, Wei Q, Tanguay RM and Wu T: Overexpressed heat shock protein 70 protects cells against DNA damage caused by ultraviolet $\mathrm{C}$ in a dose-dependent manner. Cell Stress Chaperones 11: 162-169, 2006.

11. Salvesen GS and Duckett CS: IAP proteins: blocking the road to death's door. Nat Rev Mol Cell Biol 3: 401-410, 2002.

12. Felix RS, Colleoni GW, Caballero OL, Yamamoto $M$ Almeida MS, Andrade VC, Chauffaille MdeL, Silva WA Jr, Begnami MD, Soares FA, et al: SAGE analysis highlights the importance of p53csv, ddx5, mapkapk2 and ranbp2 to multiple myeloma tumorigenesis. Cancer Lett 278: 41-48, 2009.

13. Neve RM, Chin K, Fridlyand J, Yeh J, Baehner FL, Fevr T, Clark L, Bayani N, Coppe JP, Tong F, et al: A collection of breast cancer cell lines for the study of functionally distinct cancer subtypes. Cancer Cell 10: 515-527, 2006.

14. Ali S and Coombes RC: Endocrine-responsive breast cancer and strategies for combating resistance. Nat Rev Cancer 2: 101-112, 2002.

15. Eccles SA, Aboagye EO, Ali S, Anderson AS, Armes J, Berditchevski F, Blaydes JP, Brennan K, Brown NJ, Bryant HE, et al: Critical research gaps and translational priorities for the successful prevention and treatment of breast cancer. Breast Cancer Res 15: R92, 2013.

16. Raguz S, Adams C, Masrour N, Rasul S, Papoutsoglou P, Hu Y, Cazzanelli G, Zhou Y, Patel N, Coombes C, et al: Loss of O'-methylguanine-DNA methyltransferase confers collateral sensitivity to carmustine in topoisomerase II-mediated doxorubicin resistant triple negative breast cancer cells. Biochem Pharmacol 85: 186-196, 2013.

17. Chen J, Gomes AR, Monteiro LJ, Wong SY, Wu LH, Ng TT, Karadedou CT, Millour J, Ip YC, Cheung YN, et al: Constitutively nuclear FOXO3a localization predicts poor survival and promotes Akt phosphorylation in breast cancer. PLoS One 5: e12293, 2010

18. Tolhurst RS, Thomas RS, Kyle FJ, Patel H, Periyasamy M, Photiou A, Thiruchelvam PT, Lai CF, Al-Sabbagh M, Fisher RA, et al: Transient over-expression of estrogen receptor- $\alpha$ in breast cancer cells promotes cell survival and estrogen-independent growth. Breast Cancer Res Treat 128: 357-368, 2011.

19. Rasul S, Balasubramanian R, Filipović A, Slade MJ, Yagüe E and Coombes RC: Inhibition of gamma-secretase induces G2/M arrest and triggers apoptosis in breast cancer cells. $\mathrm{Br} \mathrm{J}$ Cancer 100: 1879-1888, 2009.

20. Yagüe E, Arance A, Kubitza L, O'Hare M, Jat P, Ogilvie CM, Hart IR, Higgins CF and Raguz S: Ability to acquire drug resistance arises early during the tumorigenesis process. Cancer Res 67: 1130-1137, 2007.

21. Filipović A, Gronau JH, Green AR, Wang J, Vallath S, Shao D, Rasul S, Ellis IO, Yagüe E, Sturge J, et al: Biological and clinical implications of nicastrin expression in invasive breast cancer. Breast Cancer Res Treat 125: 43-53, 2011.
22. Vichai V and Kirtikara K: Sulforhodamine B colorimetric assay for cytotoxicity screening. Nat Protoc 1: 1112-1116, 2006.

23. Yague E, Armesilla AL, Harrison G, Elliott J, Sardini A, Higgins CF and Raguz S: P-glycoprotein (MDR1) expression in leukemic cells is regulated at two distinct steps, mRNA stabilization and translational initiation. J Biol Chem 278: 10344-10352, 2003.

24. Raguz S, De Bella MT, Slade MJ, Higgins CF, Coombes RC and Yagüe E: Expression of RPIP9 (Rap2 interacting protein 9) is activated in breast carcinoma and correlates with a poor prognosis. Int J Cancer 117: 934-941, 2005.

25. Almeida B, Silva A, Mesquita A, Sampaio-Marques B, Rodrigues F and Ludovico P: Drug-induced apoptosis in yeast. Biochim Biophys Acta 1783: 1436-1448, 2008.

26. Sun JM, Spencer VA, Li L, Yu Chen H, Yu J and Davie JR Estrogen regulation of trefoil factor 1 expression by estrogen receptor alpha and Sp proteins. Exp Cell Res 302: 96-107, 2005.

27. Nachmias B, Ashhab Y and Ben-Yehuda D: The inhibitor of apoptosis protein family (IAPs): an emerging therapeutic target in cancer. Semin Cancer Biol 14: 231-243, 2004.

28. Wang S, Bai L, Lu J, Liu L, Yang CY and Sun H: Targeting inhibitors of apoptosis proteins (IAPs) for new breast cancer therapeutics. J Mammary Gland Biol Neoplasia 17: 217-228, 2012.

29. Hanahan D and Weinberg RA: Hallmarks of cancer: the next generation. Cell 144: 646-674, 2011.

30. Garber ME, Troyanskaya OG, Schluens K, Petersen S, Thaesler Z, Pacyna-Gengelbach M, van de Rijn M, Rosen GD, Perou CM, Whyte RI, et al: Diversity of gene expression in adenocarcinoma of the lung. Proc Natl Acad Sci USA 98: 13784-13789, 2001.

31. Lu KH, Patterson AP, Wang L, Marquez RT, Atkinson EN, Baggerly KA, Ramoth LR, Rosen DG, Liu J, Hellstrom I, et al: Selection of potential markers for epithelial ovarian cancer with gene expression arrays and recursive descent partition analysis. Clin Cancer Res 10: 3291-3300, 2004

32. Skrzypczak M, Goryca K, Rubel T, Paziewska A, Mikula M, Jarosz D, Pachlewski J, Oledzki J and Ostrowski J: Modeling oncogenic signaling in colon tumors by multidirectional analyses of microarray data directed for maximization of analytical reliability. PLoS One 5: e13091, 2010.

33. Molinari AM, Bontempo P, Schiavone EM, Tortora V, Verdicchio MA, Napolitano M, Nola E, Moncharmont B, Medici N, Nigro V, et al: Estradiol induces functional inactivation of p53 by intracellular redistribution. Cancer Res 60: 2594-2597, 2000

34. Fernández-Cuesta L, Anaganti S, Hainaut P and Olivier M: Estrogen levels act as a rheostat on p53 levels and modulate p53-dependent responses in breast cancer cell lines. Breast Cancer Res Treat 125: 35-42, 2011.

35. Somaï S, Chaouat M, Jacob D, Perrot JY, Rostène W, Forgez $P$ and Gompel A: Antiestrogens are pro-apoptotic in normal human breast epithelial cells. Int J Cancer 105: 607-612, 2003.

36. Zhang GJ, Kimijima I, Onda M, Kanno M, Sato H, Watanabe T, Tsuchiya A, Abe R and Takenoshita S: Tamoxifen-induced apoptosis in breast cancer cells relates to down-regulation of bcl-2, but not bax and bcl-X(L), without alteration of $\mathrm{p} 53$ protein levels. Clin Cancer Res 5: 2971-2977, 1999.

37. Potting C, Tatsuta T, König T, Haag M, Wai T, Aaltonen MJ and Langer T: TRIAP1/PRELI complexes prevent apoptosis by mediating intramitochondrial transport of phosphatidic acid. Cell Metab 18: 287-295, 2013.

38. Cain K: Chemical-induced apoptosis: formation of the Apaf-1 apoptosome. Drug Metab Rev 35: 337-363, 2003. 